\title{
DEVELOPMENT AND APPLICATION OF AN OPTIMIZATION PROTOCOL FOR DIRECTIONAL SOLIDIFICATION: INTEGRATING FUNDAMENTAL THEORY, EXPERIMENTATION AND MODELING TOOLS
}

\author{
Jonathan D. Miller ${ }^{1}$ and Tresa M. Pollock ${ }^{2}$ \\ ${ }^{1}$ Air Force Research Laboratory, Materials and Manufacturing Directorate; 2230 Tenth St; Wright Patterson AFB, OH 45433, USA \\ ${ }^{2}$ Materials Department, University of California Santa Barbara; Santa Barbara, CA 93106, USA
}

Keywords: Directional Solidification, Solidification Modeling, Liquid Metal Cooling

\begin{abstract}
A protocol for identifying the preferred process conditions for directional solidification has been developed using the axial thermal gradient at the surface of the casting during solidification. Solidification modeling has been utilized to predict local thermal conditions during solidification for a broad range of geometrical configurations, alloy compositions and heat-extraction conditions. Three different mold configurations were evaluated for three alloy compositions using both conventional and high-gradient directional solidification processes. The high-gradient directional solidification process investigated was the Liquid Metal Cooling (LMC) process that utilizes a liquid-metal coolant in the cold zone of the directional-solidification furnace. Process conditions associated with the development of dendritic structure and solidification defects have been analyzed in detail for each configuration. Classical defect maps have been extended to consider the important effects of solid-liquid interface curvature. The utilization of the surface maximum axial thermal gradient as a means to identify preferred processing conditions is applicable to a range of solidification conditions, and accounts for changes in casting geometry, alloy composition or degree of heat-extraction. Experiments have been conducted to validate model predictions and improve the understanding of the role of solid-liquid interface curvature on dendrite-growth morphology. The optimization technique has been demonstrated for an atypical casting configuration and applied to a complex geometry, in which timedependent process conditions were required to maintain desired single-crystal growth.
\end{abstract}

\section{Introduction}

Columnar-grained and single-crystal nickel-base superalloys have been used for high-pressure turbine blades for over five decades due to their exceptional high-temperature performance. These materials are currently produced by directional solidification via the Bridgman process (Figure 1a) [1]. During this process, an investment casting is withdrawn from the hot section to the cold section of a vacuum furnace. The process relies on radiation heating in the hot zone and radiation cooling in the cold zone to maintain a relatively flat solid-liquid interface that proceeds along the casting as it is withdrawn in the furnace. For large or complex geometries it is difficult to maintain a planar-growth front and a small processing window exists due to breakdown in singlecrystal growth due to inefficient radiation cooling.

The Liquid Metal Cooling (LMC) directional-solidification process has been developed and addresses the need for enhanced heat extraction (Figure 1b). The process utilizes a liquid-metal coolant in the cold-section of the furnace to efficiently extract heat from the casting [2]. Production implementation of the LMC process for blades provided a 2-10x increase in growth rate [3]. The process has demonstrated 3-9x increase in cooling rate, corresponding to a $50 \%$ reduction in microstructure scale with reduced defect occurrence [4-17]. The refined microstructure has resulted in a 3-7x improvement in fatigue life on simple geometries [17]. However, the enhanced lateral heat extraction from the surface of the mold can lead to solidification-front curvature, which promotes the formation of misoriented grains [15]. The effect of solid-liquid interface curvature on dendrite morphology and on the propensity to form defects is not well understood.
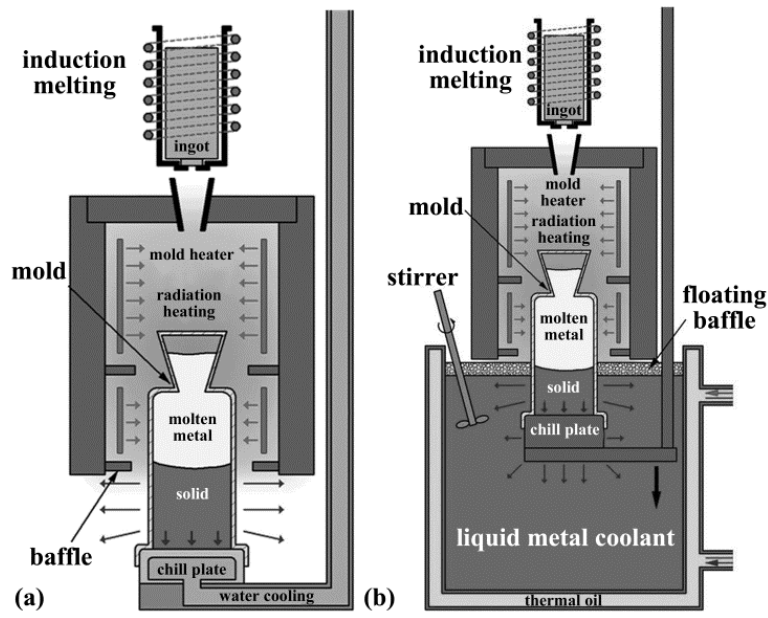

Figure 1. Schematics of the (a) Bridgman and (b) LMC processes [7].

Previous LMC experiments have observed the formation of a dendrite morphology referred to as lateral growth (Figure 2) [15]. It was demonstrated that lateral growth occurred when the local thermal gradient was better aligned with a secondary dendrite arm than the primary dendrite arm, causing the secondary arm to grow faster than and overgrow primary dendrite arms well-aligned with the withdrawal direction [15]. The lateral growth regimes were large, in some cases with dendrites completely growing from the surface to the center of cylindrical bars [15]. 


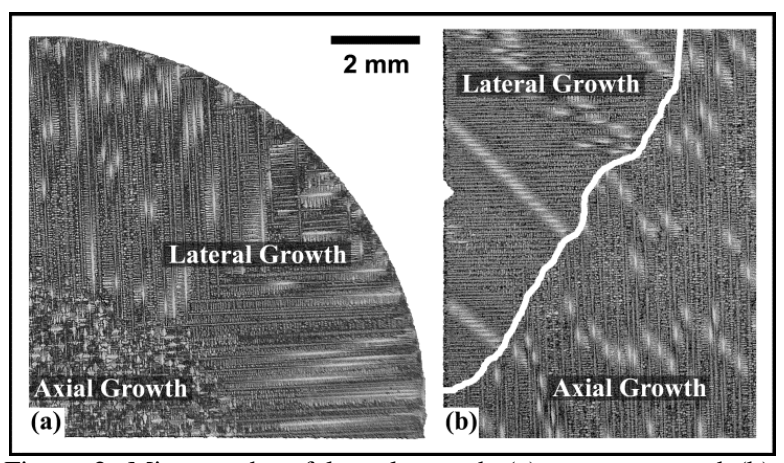

Figure 2. Micrographs of lateral growth (a) transverse and (b) longitudinal to the growth direction [15]. Note: A white line has been used to highlight the boundary between the dendrite morphologies in (b).

Several previous experimental investigations have been conducted to develop relationships between macroscopic thermal conditions and dendrite morphology [18-22]. The dendrite morphology has been associated with the thermal gradient during solidification and the solidification rate (Figure 3). Regimes of axial growth, freckle formation, nucleated grains and complete breakdown have been defined. The underlying mechanisms that control microstructure scale have also been defined in similar analyses that demonstrate refinement with increased thermal gradient $(G)$ and solidification rate $(V)$. Due to limitations of the experimental techniques, an assumption has been made that the solid-liquid interface is flat [18-20]. Thus, classical defect maps do not currently account for curvature of the solid-liquid interface. In addition, the dendrite-growth models are incapable of incorporating curvature into predictions of microstructure scale.

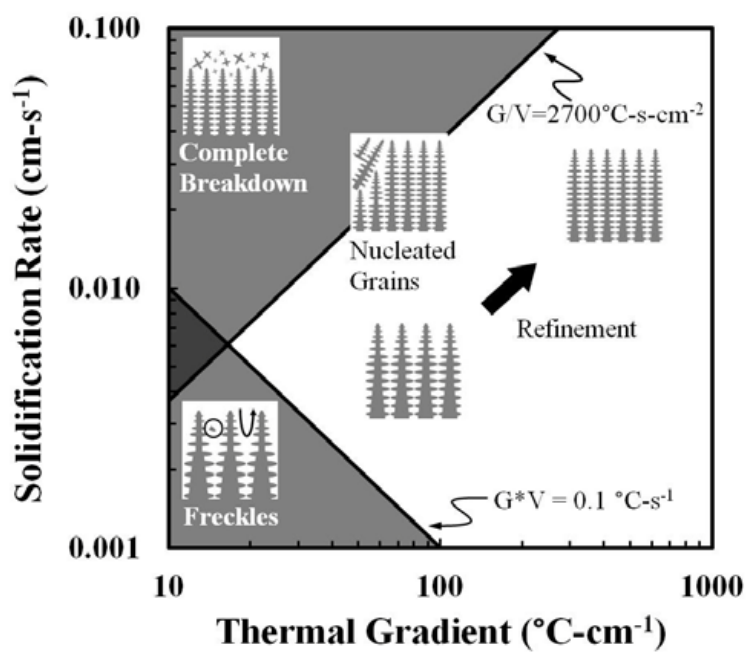

Figure 3. Adapted map of classical defect-formation criteria with superimposed schematics of dendrite morphology for directional solidification [18-19].

Continuum solidification models have been used to develop trends in directional-solidification behavior based on broad changes in processing conditions [11-16,23-24]. The ability for process simulations to accurately predict changes in local thermal conditions during solidification due to subtle variations in process conditions has only recently been possible, especially for relevant geometrical configurations. State-of-the-art solidification models are capable of utilizing time-, temperature- and locationdependent material properties and boundary conditions that enable accurate predictions of solidification behavior. However, continued advances such as predicting stresses and incorporating pressure predictions into boundary conditions are still desired.

The characterization of local thermal conditions as a function of withdrawal rate and furnace temperature for directional solidification is challenging and typically requires multiple casting trials. The process parameters are generally dependent on alloy composition and casting geometry, complicating the determination of preferred processing conditions. The intent of this research is to utilize fundamental solidification theory and solidification modeling to develop a geometry-independent metric that can be applied to specific casting geometries and alloys to identify preferred processing conditions for a broad range of solidification conditions that include curvature of the solid-liquid interface. Detailed casting experiments have been employed to validate solidification-modeling predictions.

\section{Procedure}

\section{Experimental Methods}

Casting trials using an ALD directional-solidification furnace were utilized to support model validation and verification. Additional details of the furnace have been documented elsewhere [15]. This furnace is capable of directional solidification in Bridgman and LMC modes, providing a broad range of heat extraction conditions. A key aspect of the LMC furnace configuration is the utilization of a floating ceramic baffle atop the coolant, in this case tin, in order to shield the coolant from radiation heating via the mold heater. The floating baffle was 1.5 $\mathrm{cm}$ thick and comprised of mullite beads $3.2 \mathrm{~mm}$ in diameter.

The molds for each casting configuration were supplied by PCC Airfoils. A seed and grain selector were utilized for all castings to ensure single-crystal growth of the proper crystallographic orientation. Type $\mathrm{C}$ thermocouples were utilized to profile the hot zone of the furnace, obtain cooling curves at the mold-metal interface and validate solidification predictions. A hole was drilled through the mold for proper positioning of the thermocouples at the mold-metal interface. The nominal mold thickness was $0.8 \mathrm{~cm}$. Three commercial single-crystal superalloys were utilized: René N4, René N5 and CMSX-486. The nominal alloy compositions are summarized in Table 1.

Table 1. Compositions of investigated alloys (in wt. pct.).

\begin{tabular}{ccccccccccccc}
\hline Alloy & $\mathrm{Cr}$ & $\mathrm{Co}$ & $\mathrm{Mo}$ & $\mathrm{W}$ & $\mathrm{Ta}$ & $\mathrm{Re}$ & $\mathrm{Al}$ & $\mathrm{Ti}$ & $\mathrm{Hf}$ & $\mathrm{C}$ & Other & $\mathrm{Ni}$ \\
\hline René N4 & 9.8 & 7.5 & 1.5 & 6.0 & 4.8 & & 4.2 & 3.5 & 0.15 & 0.05 & $0.5 \mathrm{Nb}$ & bal \\
René N5 & 7.0 & 7.5 & 1.5 & 5.0 & 6.5 & 3.0 & 6.2 & & 0.15 & 0.05 & $0.01 \mathrm{Y}$ & bal \\
CMSX-486 & 5.0 & 9.3 & 0.7 & 8.6 & 4.5 & 3.0 & 5.7 & 0.7 & 1.2 & 0.07 & $0.015 \mathrm{~B}$ & bal \\
\hline
\end{tabular}

Three geometrical configurations were investigated: (a) a 5-bar cluster-mold cast of René N5 [16], (b) a specially designed mold to investigate lateral dendrite growth using alloys CMSX-486 and René N4 [15] and (c) a tri-crystal ring segment cast using alloy CMSX-486 (Figure 4) [13-15]. 
(a)

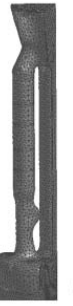

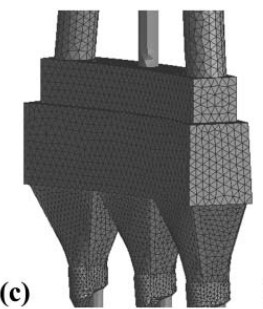

(d)

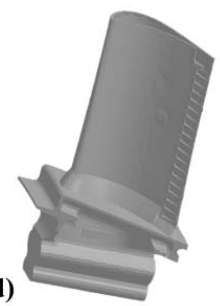

Figure 4. Casting geometries: (a) rotationally-symmetric section of a 5-bar mold, (b) rotationally-symmetric section of the lateralgrowth mold, (c) tri-crystal and (d) airfoil.

The 5-bar cluster consisted of 5 bars evenly distributed around a support post with the center of the bar positioned at a radius of 5.1 $\mathrm{cm}$ from the center of the cluster. Each bar was $15.9 \mathrm{~cm}$ long and $1.6 \mathrm{~cm}$ in diameter. The alloy utilized for this investigation was René N5. The withdrawal rates investigated were $3.4 \mathrm{~mm}-\mathrm{min}^{-1}$ for the Bridgman process and 8.5, 12.7 and $21.2 \mathrm{~mm}^{-\mathrm{min}^{-1}}$ for the LMC process.

The lateral-growth casting (Figure 4b) utilized the 5-bar cluster previously described with a modification to increase the relative lateral heat extraction. Two patches of woven alumina fabric, herein referred to as "wrap", were cut to a width of $2.5 \mathrm{~cm}$, wet with alumina cement and affixed to the exterior of the mold encircling the bar and centered at 4.5 and $10.8 \mathrm{~cm}$ above the bottom of the bar. Each bar was modified with a different wrap thickness of 0.5, 1.0 and $1.4 \mathrm{~cm}$, including one bar with no additional wrap and one replicate bar with a wrap thickness of 1.0 $\mathrm{cm}$. The additional wrap was intended to increase the lateral heat extraction just above the wrap relative to within the wrap in order to develop varying degrees of solidification-front inclination at the surface of the bar just above the wrap. Only the LMC process was evaluated for this mold configuration. Withdrawal rates of 12.7, 16.9 and $21.2 \mathrm{~mm}-\mathrm{min}^{-1}$ were investigated for alloy CMSX486, while withdrawal rates of $8.5,12.7$ and $16.9 \mathrm{~mm}-\mathrm{min}^{-1}$ were investigated for René N4.

The tri-crystal ring segment (Figure 4c) was a 30-degree segment of a full ring with a height of $2.5 \mathrm{~cm}$. The ring segment was divided into three 10-degree segments in which each 10-degree segment had a seed and grain selector from which to grow a different crystallographic orientation. The primary orientation was aligned with the axial orientation and secondary orientation with the radial orientation of the ring segment. This configuration resulted in three segmented single-crystals with crystallographic boundaries between the segments misoriented by 10 degrees. Two ring thicknesses (inner- to outer-diameter distance) were considered, designated as thick $(5.1 \mathrm{~cm})$ and thin $(1.9 \mathrm{~cm})$. The intent of this investigation was to assess the feasibility of casting multiply-seeded 'single-crystal' rings of different thicknesses using conventional and high-gradient directional-solidification processes. Each ring thicknesses was investigated for each of the Bridgman and LMC processes with a single casting trial based on process conditions determined using solidification modeling as will be described in the following section.

Castings were sectioned and prepared for metallographic analysis. The Primary Dendrite Arm Spacing (PDAS) and Secondary Dendrite Arm Spacing (SDAS) were measured according to wellaccepted techniques as described elsewhere [15]. Relative differences in PDAS and SDAS were compared with relative changes in the predicted thermal conditions of the castings.

\section{$\underline{\text { Simulation Methods }}$}

A finite-element solidification model, ProCAST ${ }^{\mathrm{TM}}$ (a trademark of ESI Group, Inc.), was utilized to predict the spatial-dependence of thermal conditions during directional solidification. Details regarding model parameters and boundary conditions have been summarized in Table 2 and fully described elsewhere [15]. For different alloys, the thermophysical properties of the metal were adjusted, which will affect the resulting thermal conditions and dendrite structure. The solidification model has been validated from four different process and mold-configuration conditions encompassing a broad range of heat-extraction conditions by comparison of thermocouple measurements at the mold-metal interface, exterior mold surface, and specific locations within the hot, cold and transition zones of the directional solidification furnace [14-15].

Table 2. Model parameters for FE-based solidification modeling.

\begin{tabular}{|c|c|c|c|}
\hline \multicolumn{2}{|c|}{ Thermophysical Properties } & \multicolumn{2}{|c|}{ Interface Heat Transfer Coefficients } \\
\hline & & Superalloy-Ceramic & $* 750 \mathrm{~W} / \mathrm{m}^{2} \mathrm{~K}$ \\
\hline & & Ceramic-Cera & $500 \mathrm{~W} / \mathrm{m}^{2} \mathrm{~K}$ \\
\hline & $6.4 \mathrm{~mm}$ & Ceramic & $4000 \mathrm{~W} / \mathrm{m}^{2} \mathrm{~K}$ \\
\hline \multicolumn{2}{|c|}{ Boundary Conditions } & \multicolumn{2}{|c|}{ Run Parameters } \\
\hline & 0.4 & Im Time Step & ** \\
\hline & 0.2 & Spatial Step & $0.5 \mathrm{r}$ \\
\hline Temperature & $250^{\circ} \mathrm{C}$ & Critical Fraction Soli & 0.4 \\
\hline
\end{tabular}

*Initial value reported, exponential decay to $100 \mathrm{~W} / \mathrm{m}^{2} \mathrm{~K}$ during solidification

**S Scaled to provide equal withdrawal distance $(0.4 \mathrm{~mm})$ between steps

A key boundary condition for the LMC process simulations was location-dependent heat-transfer conditions at the surface of the mold that varied whether the particular node was in the mold heater, floating baffle or liquid coolant regions of the furnace (Table 3). Initially, the entire mold was in the mold-heater region and radiation heating occurred from the heating elements through application of an emissivity on the surface of the mold. During withdrawal, each mold-surface node passed through the floating baffle, during which the emissivity was turned off and ceramicceramic interface heat transfer coefficient applied. The ambient temperature was assigned according to the thermal gradient occurring through the thickness of the floating baffle. Finally, as each node passed from the floating baffle into the coolant, the interface heat transfer coefficient was increased to a value consistent with a liquid metal-ceramic interface and the ambient temperature was set to the experimentally-observed temperature of the tin bath.

Table 3. Location-dependent boundary conditions to simulate interactions of floating baffle and coolant with mold.

\begin{tabular}{cccc}
\hline & & $\begin{array}{c}\text { Interface Heat } \\
\text { Transfer Coefficient } \\
\left(\mathrm{W} / \mathrm{m}^{2} \mathrm{~K}\right)\end{array}$ & $\begin{array}{c}\text { Ambient } \\
\text { Lemperature }\left({ }^{\circ} \mathrm{C}\right)\end{array}$ \\
\hline Above Baffle & 0.4 & 0 & ---- \\
Within Baffle & 0 & 500 & $1100-325$ \\
Below Baffle & 0 & 4000 & 250 \\
\hline
\end{tabular}


A simulated parametric analysis was conducted for each casting configuration to evaluate local thermal conditions as a function of withdrawal rate conditions (Figure 5 and Table 4). The sensitivity of thermal conditions to bar thickness, mold-heater temperature and alloy composition was also evaluated for the 5-bar cluster configuration (Figure 5). Overall, 200 simulations for the 5-bar cluster, 24 simulations for the lateral-growth cluster and 20 simulations for the tri-crystal configuration were analyzed.

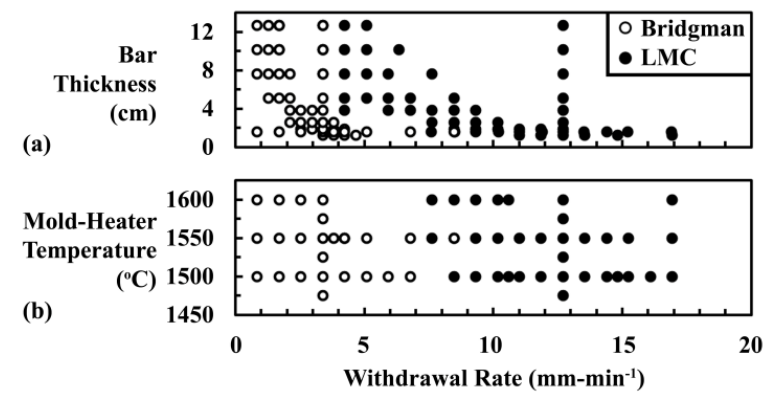

(c)

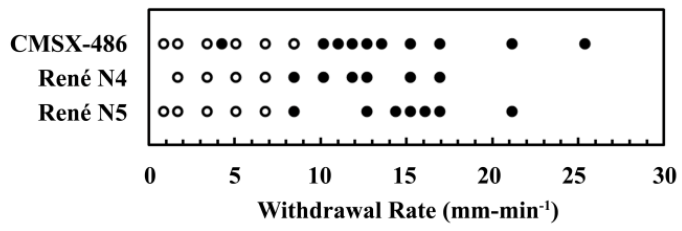

Figure 5. Simulated two-parameter analyses for 5-bar cluster, withdrawal rate and (a) bar thickness, (b) mold-heater temperature and (c) alloy.

Table 4. Simulated withdrawal rates for the lateral-growth and tricrystal ring-segment casting configuration.

\begin{tabular}{lccc}
\hline Configuration & Alloy & Process & Withdrawal Rates $\left(\mathrm{mm}-\mathrm{min}^{-1}\right)$ \\
\hline \multirow{2}{*}{ lateral growth } & CMSX-486 & LMC & $12.7,16.9,21.2$ \\
& Rene N4 & LMC & $8.5,12.7,16.9$ \\
\hline \multirow{2}{*}{ thick tri-crystal } & \multirow{2}{*}{ CMSX-486 } & Bridgman & $0.8,1.7,2.5,3.4,4.2$ \\
& & LMC & $4.2,5.9,6.8,7.6,9.3$ \\
\hline \multirow{2}{*}{ thin tri-crystal } & \multirow{2}{*}{ CMSX-486 } & Brudgman & $1.7,2.5,3.4,4.2,5.1$ \\
& & LMC & $5.1,6.8,8.5,10.2,11.9$ \\
\hline
\end{tabular}

The thermal conditions examined include (1) the thermal gradient in the withdrawal direction (herein referred to as the axial thermal gradient), (2) the solidification rate in the withdrawal direction (herein referred to as the axial solidification rate, (3) the cooling rate from liquidus to solidus, (4) the inclination angle of the solidliquid-interface (relative to horizontal), (5) the solid-liquidinterface position relative to the transition region in the furnace (the top of the fixed baffle in Bridgman and top of the floating baffle in the LMC process, herein referred to as the relative position, Figure 6). One node at the surface and center of each casting configuration was evaluated: at the mid-height of the 5-bar cluster, just above the wrap of the lateral-growth casting and at the mid-height of the tri-crystal ring segment. The values of the thermal conditions were analyzed with changes in process conditions.
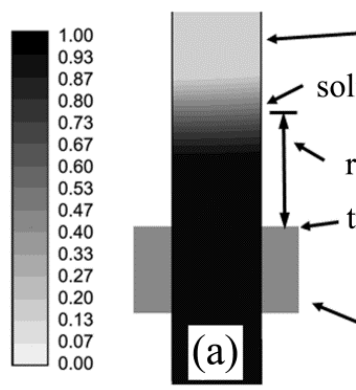

casting

Figure 6. Schematic of fraction-solid contours depicting the solidliquid interface position relative to the transition region in the furnace for the (a) Bridgman and (b) LMC processes.

The PDAS $\left(\lambda_{1}\right)$ and SDAS $\left(\lambda_{2}\right)$ were predicted from the local thermal conditions according to well-accepted dendrite-growth models, Equations 1 and 2, respectively [19,20].

$$
\begin{gathered}
\lambda_{1}=A_{1} G_{Z}^{-1 / 2} V^{-1 / 4} \\
\lambda_{2}=A_{2}(L)^{-1 / 3}
\end{gathered}
$$

In these equations, $A_{1}$ and $A_{2}$ are coefficients dependent on alloy composition, $G_{Z}$ is the axial thermal gradient, $V$ is the solidification rate and $L$ is the cooling rate. The original development of the dendrite growth model for primary dendrite arm spacing assumed a flat solid-liquid interface and utilized the thermal gradient and alloy melting range to approximate the length of the parabolic dendrite. Thus, when extending the model to curved solid-liquid interfaces, only the thermal gradient parallel to the dendrite growth direction - the axial thermal gradient should be considered. Measured PDAS and SDAS were compared to predicted PDAS and SDAS based on local thermal conditions.

Thermal conditions across a range of alloy and casting configurations were analyzed to identify geometry-independent criteria for optimization of the casting process. Using these criteria, parametric studies of process conditions were then employed to define a preferred process condition for each casting configuration. The optimization approach maximized heat extraction while also limiting the probability for the formation of solidification defects for a given casting geometry and alloy. The range of alloy compositions and geometrical configurations allowed for generalization of the optimization approach. The thermal conditions were also compared to classical solidification maps and defect criteria for each casting configuration to assess the validity of the data from experience-based processing maps [18-19].

Finally, the optimization protocol was applied to a complex airfoil geometry (Figure 4). Directional solidification of a 4-airfoil cluster of CFM56-3 airfoils was simulated for the LMC process. A range of constant withdrawal rates were evaluated: 6.4, 8.5, 10.6, 12.7, 14.8, 16.9, 19.1 and $23.3 \mathrm{~mm}-\mathrm{min}^{-1}$. Thermal conditions from nodes throughout the casting were analyzed with respect to the axial position of the node along the blade axis. A technique to define a time-dependent withdrawal rate from a simulated parametric analysis was demonstrated. 
Results

\section{Case A: Five-Bar Cluster}

Predictions of local thermal conditions at the surface of a bar at its mid-height from a simulated parametric analysis of the directional solidification process indicated that a maximum axial thermal gradient was achieved at an intermediate withdrawal rate (Figure 7). The axial thermal gradient is an important thermal metric during directional solidification because the primary dendrite arm spacing is controlled by the thermal gradient in the growth (axial) direction. As the withdrawal rate increased, the solidification front lowered further from the hot zone of the furnace. At a withdrawal rate of $11.9 \mathrm{~mm}-\mathrm{min}^{-1}$, the solidification front was 0.2 $\mathrm{cm}$ above the floating baffle, in the transition region between the hot and cold zones of the furnace, and a maximum axial thermal gradient of $82{ }^{\circ} \mathrm{C}-\mathrm{cm}^{-1}$ was achieved at the surface of the casting (Figure 7). Any further increase in the withdrawal rate caused significant curvature of the solid-liquid interface, thus reducing the axial thermal gradient. However, the axial thermal gradient in the center of the casting further increased, causing variations in the solidification conditions from the surface to the center of the casting. The cooling rate increased with increasing withdrawal rate in all cases, despite the severely inclined solid-liquid interface (Figure 7).
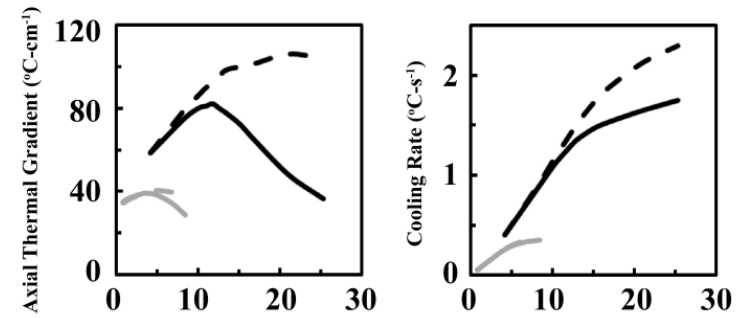

(a)

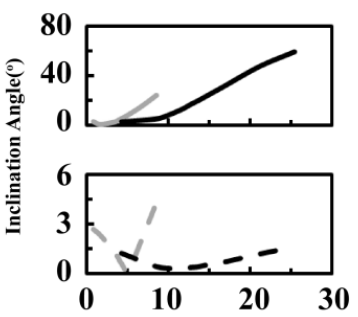

(b) Withdrawal Rate (mm-min $\left.{ }^{-1}\right)$

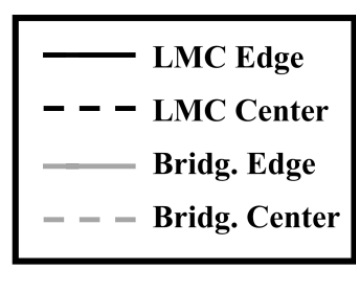

(c) Withdrawal Rate (mm- $\left.\mathrm{min}^{-1}\right)$

Figure 7. Predicted sensitivity of thermal conditions to withdrawal rate at the surface and center of the bar at its mid-height for a 5bar cluster processed via Bridgman and LMC, (a) axial thermal gradient, (b) cooling rate and (c) inclination angle.

Classical defect-formation criteria have been extended to nonaxial heat extraction during solidification by utilization of the axial thermal gradient and axial solidification rate. Comparison between experimental observations and defect criteria validated the use of the axial thermal conditions, as will be discussed in the following section. The predicted axial thermal gradient and axial solidification rate at the surface of the bar for each of the simulated castings were superimposed on the modified classical solidification maps (Figure 8). The casting conditions that achieved the maximum axial thermal gradient were within the directional growth zone of the solidification maps. An increase in withdrawal rate from this position approached the thermal conditions consistent with stray grains, in which a change in dendrite morphology from axial growth to lateral growth secondary dendrite arms over-growing well-aligned primary arms - and ultimately stray grain formation was observed. Conversely, a decrease in the withdrawal rate approached the thermal conditions consistent with freckle formation.

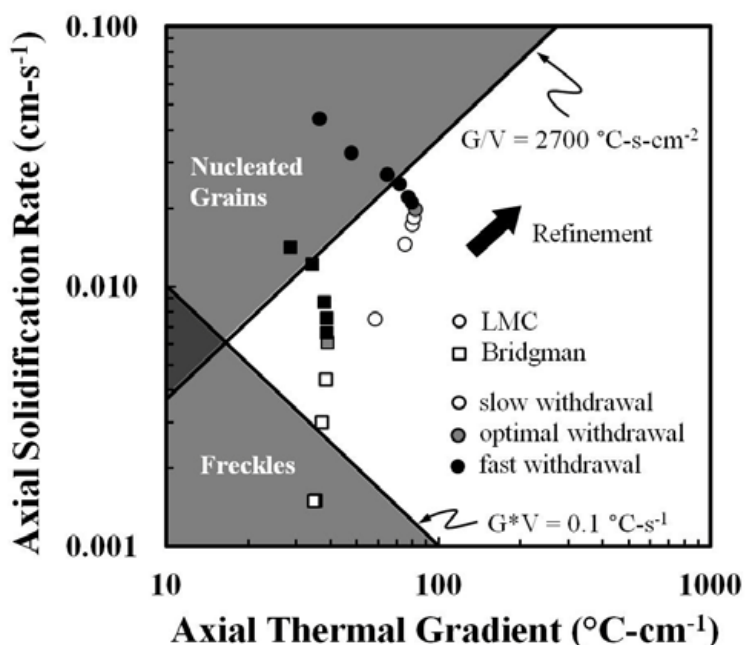

Figure 8. Defect map with superimposed predicted solidification conditions for a range of withdrawal rates. The simulated solidification condition for the preferential withdrawal rate is identified as a grey circle, while withdrawal rates slower and faster than the preferred are colored white and black, respectively.

A similar analysis was performed for bar thicknesses varying from 1.3 to $12.7 \mathrm{~cm}$ in diameter and at furnace temperatures from 1475 to $1600{ }^{\circ} \mathrm{C}$. The same trend of maximum axial thermal gradient at an intermediate withdrawal rate was observed (Figure 9a). More specifically, the maximum axial thermal gradient was achieved when the position of the solid-liquid interface at the exterior of the casting was approximately 0.5 to $1.5 \mathrm{~cm}$ and 0.2 to $0.6 \mathrm{~cm}$ above the transition region for the Bridgman and LMC processes, respectively, independent of furnace temperature or casting geometry (Figure 9b).

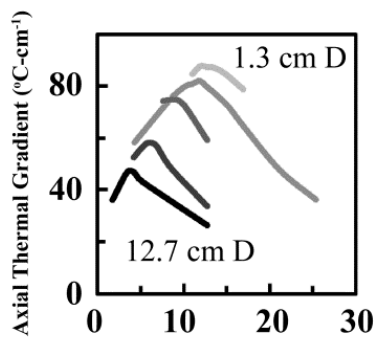

(a) Withdrawal Rate $\left(\mathrm{mm}-\mathrm{min}^{-1}\right)$

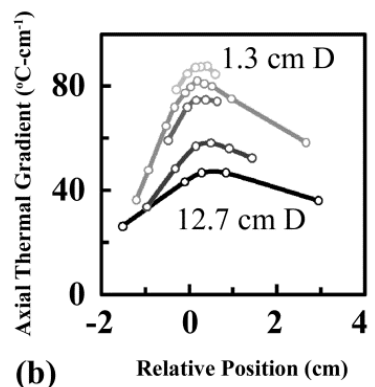

(b)
Figure 9. Predicted axial thermal gradient for a range of bar thicknesses (increasing in darkness: 1.3, 1.6, 2.5, 5.1, $12.7 \mathrm{~cm}$ diameter) cast at varying withdrawal rates plotted as a function of (a) withdrawal rate and (b) relative position. 
The thermal conditions associated with the withdrawal rate that provided the maximum axial thermal gradient for each bar thickness or mold-heater temperature mold configuration were compared and superimposed on a solidification defect map (Figure 10). Decreasing the bar thickness for either the Bridgman or LMC process refined the dendritic structure, increasing both the axial thermal gradient and axial solidification rate without proceeding to the nucleated grain regime. For very large bar thicknesses (greater than $7.5 \mathrm{~cm}$ in diameter) produced via the Bridgman process, the thermal conditions are consistent with the formation of freckles according to the defect map. However, the thicknesses in the freckle regime of Figure $\mathbf{1 0}$ were not evaluated experimentally, so the accuracy of the " $0.1{ }^{\circ} \mathrm{C}-\mathrm{s}^{-1}$ " line could not be validated. Increasing the mold-heater temperature for either the Bridgman or LMC process increased the axial thermal gradient and decreased the axial solidification rate. Based on the predictions, by increasing the mold-heater temperature, structure refinement did not occur, but the propensity to form defects decreased.

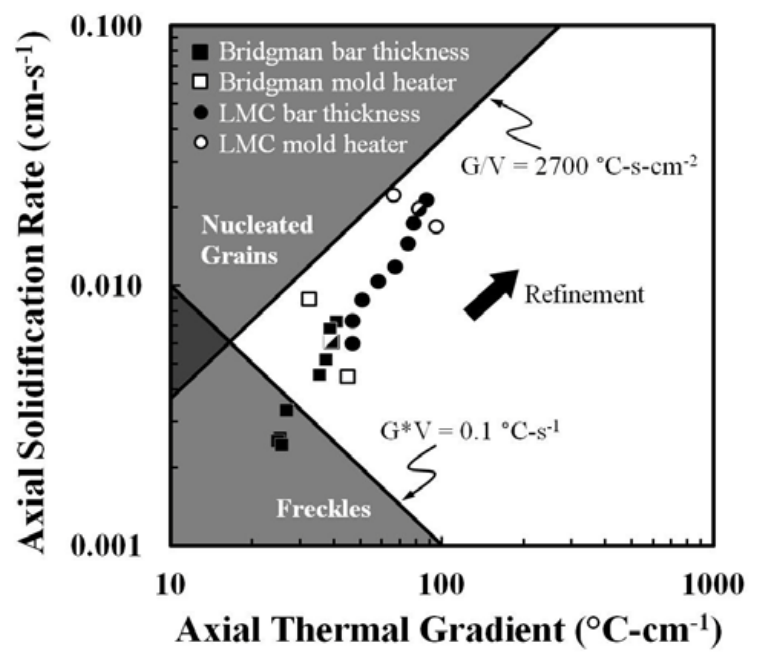

Figure 10. Defect map with predicted preferred solidification conditions for a range of bar thicknesses and mold-heater temperatures.

Overall, maximization of the axial thermal gradient required a balance of efficient heat extraction with minimal interface curvature. In addition, the same combination of relative solidliquid interface position and maximum axial thermal gradient occurred for a broad range of bar thicknesses and mold-heater temperatures. The insensitivity of the solid-liquid interface position to bar thickness and mold-heater temperature suggested that there is a desirable solidification position between the hot and cold zones of the furnace. Thus, the axial thermal gradient was utilized as the critical solidification metric and will be the focus of the remainder of this paper. Typically, the withdrawal rate is the process parameter that is varied to achieve single-crystal solidification. The approach for each casting configuration herein will be to (1) vary the withdrawal rate, (2) maximize the thermal gradient, (3) monitor the relative position of the solid-liquid interface and (4) compare findings to classical defect criteria.

Initial demonstration of the optimization approach has been developed by comparison to experimental efforts previously published [16]. Metallographic analysis of castings from a range of withdrawal rates demonstrated that a minimum primary dendrite arm spacing (PDAS) was achieved at an intermediate withdrawal rate (Figure 11) [16]. Superposition of the PDAS measurements and predicted axial thermal gradients as a function of withdrawal rate confirmed that the minimum PDAS was observed for the experimental condition that provided the predicted maximum axial thermal gradient, relative to the other experimental conditions. Therefore, not only does the optimization protocol provide conditions that balance enhanced heat extraction and solidification-front curvature, but also provide optimal refinement in dendrite structure.

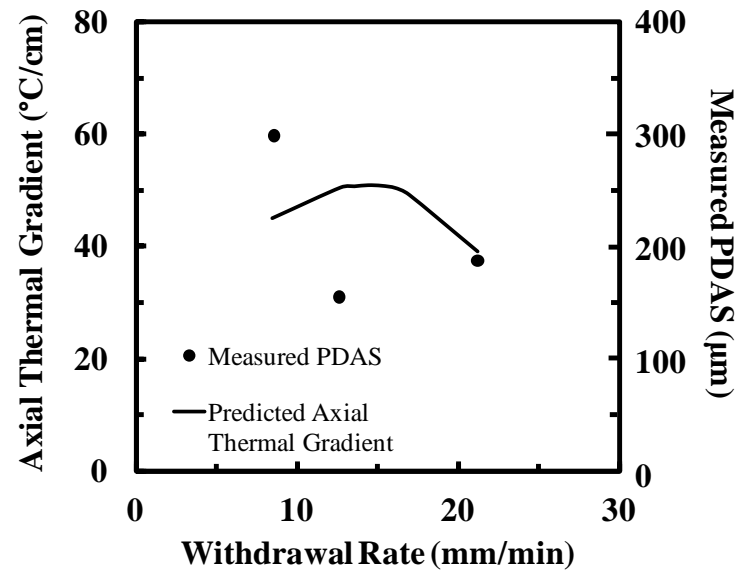

Figure 11. Comparison of predicted axial thermal gradient and measured primary dendrite arm spacing for bars composed of René N5 cast at varying withdrawal rates (measurements adopted from [16]).

\section{Case B: Lateral-Growth Casting}

Further analysis was conducted on the morphology of the solidification structure and its sensitivity to the solid-liquid interface curvature. This analysis (1) complements the previous analysis by decoupling the section thickness effect and thermal conditions with respect to dendrite-growth morphology and (2) highlights the importance of considering the axial thermal gradient and axial solidification rate in the presence of non-axial heat extraction.

As reported in the previous section, an increase in solid-liquid interface curvature results in a change in dendrite morphology from axial growth to lateral growth and ultimately strain grain formation. A previous analysis [15] developed a relationship between inclination angle and dendrite-growth morphology through LMC casting trials and simulations (Figure 12). The onset of lateral growth occurred at an inclination angle of 25 degrees, while the presence of nucleated grains was observed at inclination angles greater than 45 degrees. 


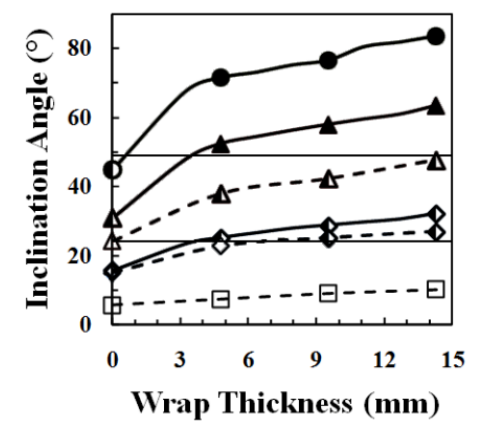

Withdrawal Rate $(\mathrm{mm} / \mathrm{min})$

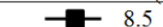

$\begin{array}{ll}\longrightarrow- & 8.5 \\ \longrightarrow & 12.7\end{array}$

$-16.9$

$-21.2$

Alloy

- CMSX-486

- - - René N4

Morphology

O Axial Growth

- Lateral Growth

- Grain Nucleation

Figure 12. Predicted inclination angle of the solid-liquid interface for a range of wrap thicknesses, withdrawal rates and alloys cast via LMC with superimposed, experimentally-observed dendrite morphology [15].

The thermal conditions from the previous lateral-growth analysis were compared to the dendrite-morphology regimes to assess whether local changes in thermal gradient and solidification rate could account for the dendrite morphology changes, rather than inclination angle as in Figure 12 (Figure 13). The curvature of the solid-liquid interface has not been previously considered with respect to the solidification defect maps, in which the total thermal gradient and solidification rate were evaluated. The onset of lateral growth occurred at an approximately constant $\mathrm{G} / \mathrm{V}$ value of approximately $3500{ }^{\circ} \mathrm{C}-\mathrm{s}-\mathrm{cm}^{-2}$, while grain nucleation was only observed at $\mathrm{G} / \mathrm{V}$ values less than $1000{ }^{\circ} \mathrm{C}-\mathrm{s}-\mathrm{cm}^{-2}$. Thus, the critical G/V value for nucleated grains has been shifted to 1000 ${ }^{\circ} \mathrm{C}-\mathrm{s}-\mathrm{cm}^{-2}$ and a new regime for lateral growth has been identified in accordance with the experimental observations. These data also indicated that lateral growth is a precursor to the presence of nucleated grains as identified on the defect map (Figure 13). No trend between $\mathrm{G} / \mathrm{V}$ and dendrite morphology was observed when the total thermal gradient and total solidification rate were considered.

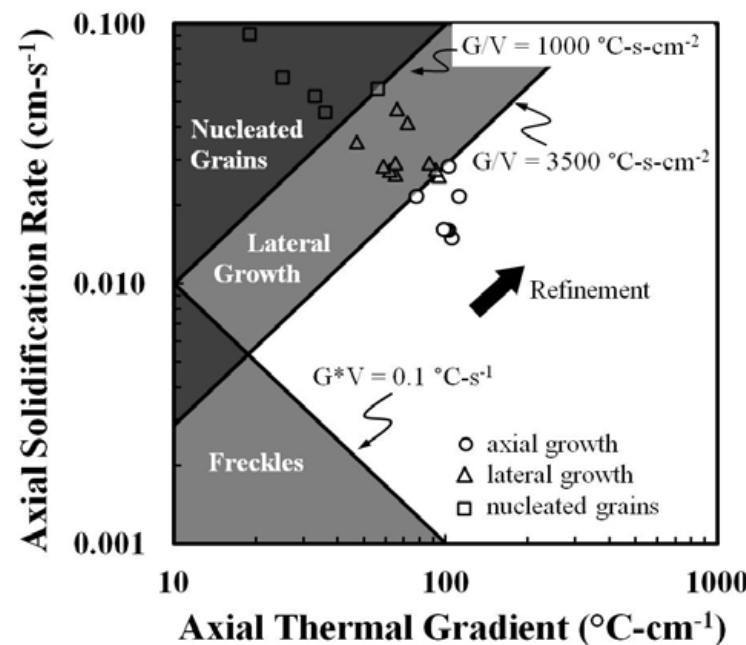

Figure 13. Defect map with superimposed predicted solidification conditions above the mold wrap for a range of wrap thicknesses and withdrawal rates to achieve a broad range of solidificationfront curvature. The onset of lateral growth and nucleated grains occur at a constant $\mathrm{G} / \mathrm{V}$ in the axial direction.

\section{Case C: Tri-Crystal Ring Segment}

In the final analysis, a more complex geometry was simulated to assess the validity of the optimization protocol to define process conditions without any experimental trials. This mold configuration was significantly different than the rotationallysymmetric, thin-section castings considered thus far. A simulated parametric analysis determined the withdrawal rate that provided the maximum axial thermal gradient, thus defining the preferred process conditions for two thicknesses and two directionalsolidification processes. Following the definition of processing conditions, casting trials were conducted at the preferred process conditions to evaluate dendrite morphology and microstructure scale. Thermocouple measurements from the casting trials were also utilized to validate model predictions.

The predicted axial thermal gradient at the surface of the tricrystal resulted in the same trend as observed in the previous two cases: a maximum axial thermal gradient was attained at an intermediate withdrawal rate for both thicknesses and both directional-solidification processes (Figure 14). As observed previously, the axial thermal gradient was less sensitive to changes in withdrawal rate for the Bridgman process. The predicted axial thermal gradient for the Bridgman-processed thick tri-crystal was near the $20{ }^{\circ} \mathrm{C}-\mathrm{cm}^{-1}$ threshold for all investigated withdrawal rates, suggesting that the thermal conditions may be insufficient for single-crystal growth.

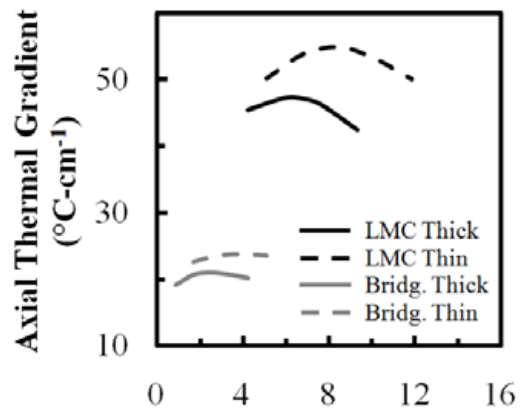

(a) Withdrawal Rate $\left(\mathrm{mm}-\mathrm{min}^{-1}\right)$

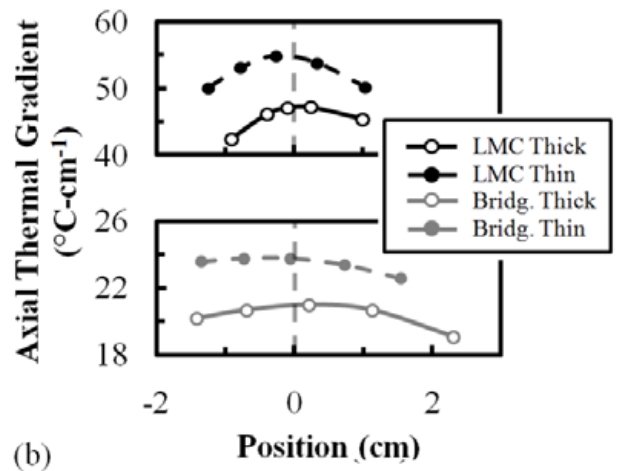

Figure 14. Predicted axial thermal gradient for the thick and thin variants of the tri-crystal ring segment processed via the Bridgman and LMC processes, (a) as a function of withdrawal rate and (b) as a function of solid-liquid interface position relative to the furnace transition region. 
In order to predict the dendrite morphology, the predicted solidification conditions were superimposed on the classical defect map (Figure 15). As identified from the axial thermal gradient predictions, the Bridgman-processed thick tri-crystal was in the regime for freckle formation. The optimal process condition for the remaining pairs of tri-crystal configurations and processes were in the dendrite-growth regime. For each configuration and directional-solidification process, the path of the predictions with increased withdrawal rate was consistent with the 5-bar cluster predictions previously plotted. Increased refinement was predicted for the thin tri-crystal casting as compared to the thick, which was consistent with the observed trend of increased refinement with reduced cross-sectional thickness due to reduced thermal mass and enhanced heat extraction. Moreover, considerable refinement was predicted for LMC-processed tri-crystals as compared to the same casting configurations processed via Bridgman.

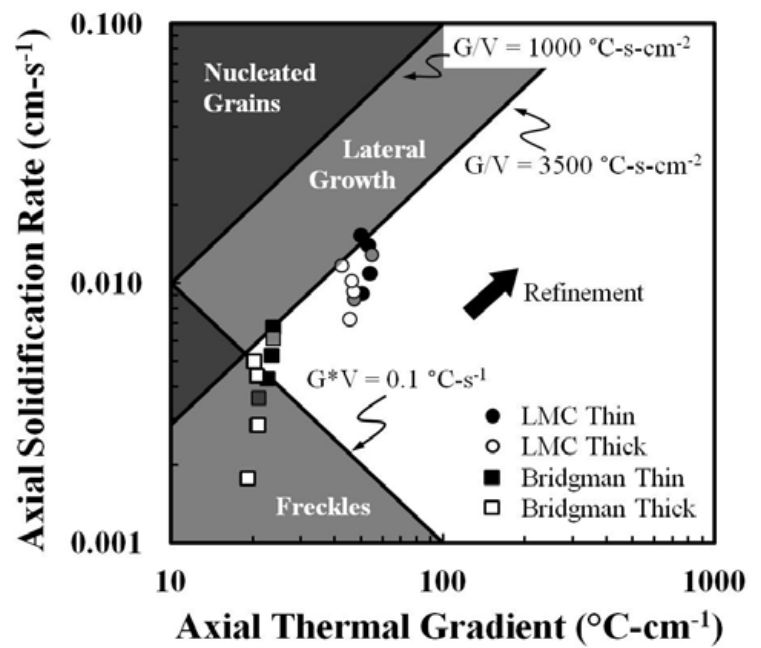

Figure 15. Defect map with superimposed predicted solidification conditions for thick $(5.1 \mathrm{~cm})$ and thin $(1.9 \mathrm{~cm})$ tri-crystal ring segments solidified via the Bridgman and LMC processes. The optimal solidification condition for each pair of casting configuration and process are shown by the shaded grey symbols.

The modeling-defined process conditions were utilized for a casting trial for each tri-crystal configuration for both directionalsolidification processes. Three thermocouples were positioned at the mold-metal interface at the surface of the casting at three different heights within the tri-crystal segment. The predicted cooling rate was within $0.05{ }^{\circ} \mathrm{C}-\mathrm{s}^{-1}$ of the cooling rate from the thermocouple measurements for each of the four different casting trials. PDAS and SDAS measurements from similar locations within each of the tri-crystal castings were compared to the predictions of PDAS and SDAS at the same locations using Eqns. (1) and (2) (Table 5) $[19,20]$. Predictions of PDAS and SDAS were within $15 \%$ of the measurements, which is considered to be within the local variability due to the measurement technique [16].
Table 5. Comparison of measured and predicted PDAS and SDAS for thick and thin tri-crystal configurations processed via the Bridgman and LMC techniques.

\begin{tabular}{llcccc}
\hline Casting Mode & \multicolumn{2}{c}{ Bridgman } & \multicolumn{2}{c}{ LMC } \\
\multicolumn{2}{l}{ Section Thickness $(\mathrm{cm})$} & 5.1 & 1.9 & 5.1 & 1.9 \\
\hline \multirow{2}{*}{ PDAS (mm) } & Measured & 406 & 334 & 248 & 187 \\
& Predicted & 420 & 334 & 210 & 158 \\
\hline \multirow{2}{*}{ SDAS (mm) } & Measured & 86 & 72 & 43 & 35 \\
& Predicted & 89 & 72 & 49 & 41 \\
\hline
\end{tabular}

Extension to Airfoil Geometries

With the relatively successful application of the protocol to the tricrystal ring segment, a cored airfoil geometry was considered. Due to the significant changes in cross-section along the axis of the blade, the optimization protocol was extended to define a time-dependent withdrawal rate by considering all casting nodes along the blades axis. The time-dependent optimization protocol has been defined as follows: (1) Conduct simulations at a broad range of constant withdrawal rates, (2) Analyze the simulations individually at all casting-surface nodes for the position of the solid-liquid interface relative to the transition region in the furnace as a function of height along the blade, (3) Generate a contour plot of relative solid-liquid interface position as a function of axial position along the blade and withdrawal rate, (4) Determine withdrawal rate as a function of time by maintaining the solid-liquid interface at a specified relative position along the length of the casting.

This protocol was applied to a selected high-pressure turbine blade geometry with the following results. The withdrawal rate has been normalized by a withdrawal rate initially estimated to provide a PDAS of $200 \mu \mathrm{m}$. The predicted relative position of the solid-liquid interface for each incremental distance along the withdrawal direction was determined for a constant withdrawal rate simulation, Figure 16. Every node at the casting surface was included in the statistical analysis (step 2), but only the average value for each $0.1 \mathrm{~cm}$ "bin" was used for the contour plot (step 3). An increase in the relative position is observed in transitioning from the relatively thick cross-section of the ramp to the relatively thin airfoil walls. A decrease in the relative position is observed as the solidification front approaches the platform in the airfoil and continues to decrease through the platform. The previous analyses indicated that a maximum axial thermal gradient occurred at a relative position of $0.5 \mathrm{~cm}$ above the floating baffle for Figure 16. Thus, the normalized withdrawal rate of 1.0 resulted in a relative position that was too high within the ramp and airfoil regions of the casting, suggesting that an increase in the withdrawal rate is required for optimal processing conditions in these regions. 


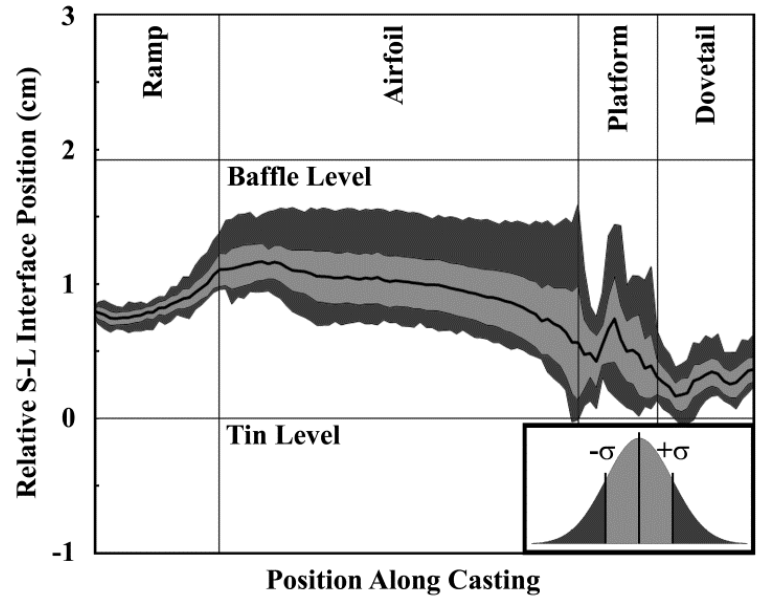

Figure 16. Solid-liquid interface position at the casting surface relative to the free surface of the coolant as a function of distance along the casting for a constant normalized withdrawal rate of 1.0.

After the analysis of each constant-withdrawal-rate simulation was completed, the data was combined onto a contour plot, Figure 17. The average value of the relative position was utilized to generate the contours. In order to maintain a relative position of $0.5 \mathrm{~cm}$ throughout the entire casting, a normalized withdrawal rate increasing from 1.2 to 1.5 in the ramp region and decreasing from 1.5 to 1.1 in the airfoil region is required, which is consistent with the analysis of Figure 16, that the normalized withdrawal rate of 1.0 is too slow to provide the optimal position.

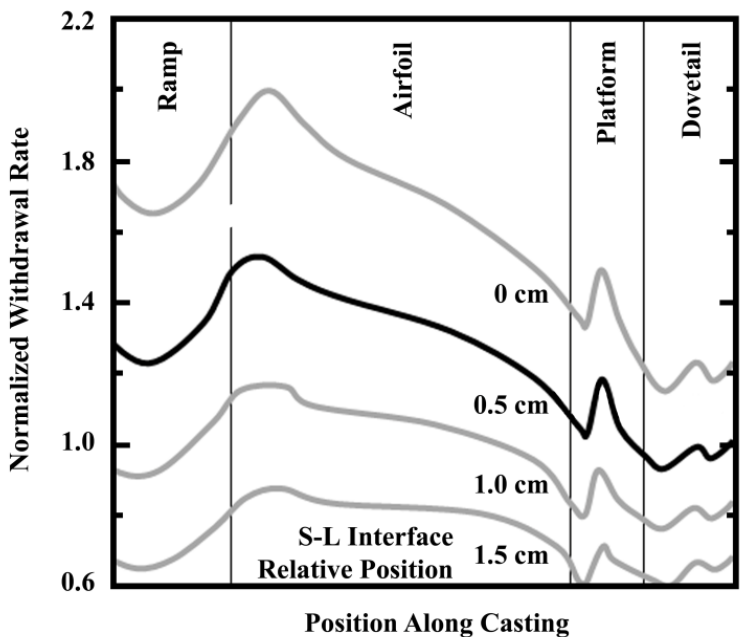

Figure 17. Constant solid-liquid interface position contours as a function of position along the casting and normalized withdrawal rate. The solid black curve is the optimal relative position.

This analysis has demonstrated that it is feasible to define a timedependent withdrawal rate using only solidification-modeling predictions, and that the modeling approach is sufficiently robust to compensate for local variability based on complex geometry. However, validation that the model-defined processing conditions achieve better solidification conditions than experience-based processing conditions from industry is ongoing.

\section{Discussion}

Key findings from this research include (1) the development and application of the maximum axial thermal gradient as a thermal metric for optimization of processing conditions for single-crystal dendrite growth, (2) an improved understanding of the effect of solid-liquid interface curvature on dendrite-growth morphology and (3) the extension of defect-formation criteria to account for curvature of the solid-liquid interface. The implications of these findings on the processing of single-crystal nickel-base superalloys are discussed in this section

The utilization of the axial thermal gradient for optimization of the directional solidification conditions provided a balance of high thermal gradient and reduced solid-liquid interface curvature. The predicted optimal solidification conditions resided within the dendrite growth regime of the classical dendrite morphology maps (Figures 8, 10 and 15). Furthermore, a correspondence between maximum axial thermal gradient and solid-liquid interface position relative to the transition region of the furnace indicated that the appropriate thermal metric was determined, since it is generally accepted that optimal directional solidification occurs when the solid-liquid interface is in the transition region of the furnace (Figures $9 \mathrm{~b}$ and $14 \mathrm{~b}$ ). This correspondence reduced the number of simulations required to determine the optimal withdrawal rate, since there is a well-established relationship between solid-liquid interface position and withdrawal rate: increasing withdrawal rate decreases relative position. Since this approach is effective for two very different processes, Bridgman and LMC, it is likely to translate to other furnace designs; this remains to be demonstrated.

The application of the optimization technique to a complex turbine-blade geometry resulted in time-dependent changes in withdrawal rate, to accommodate the changes in cross-sectional area at the solid-liquid interface. In addition, the minimum relative position for each incremental distance along the length of the blade was compared to the metal-coolant level to ensure that the relative position across the entire surface was never below the tin level due to the sharp decay in thermal conditions below the surface of the metal coolant. Microstructure scale and variability measurements from casting trials using this time-dependent withdrawal rate are needed to confirm the validity of the optimization approach. Overall, the most effective means to demonstrate the utility of the optimization protocol is to compare the results from the optimization analysis to either historical experience from industry or thorough experimental investigations such as in Figure 11.

The experiments and simulations of solidification in the lateral growth mold demonstrated the need to understand the role of solid-liquid interface curvature on dendrite morphology. Through comparison of experimentally observed dendrite morphology and predicted solidification conditions, the dendrite-morphology maps were extended to account for solid-liquid interface curvature through utilization of the axial thermal gradient and axial solidification rate. The approximation that the solid-liquid interface is flat and perpendicular to the withdrawal direction may not be valid, even for simple bar-mold geometries as indicated by Figure 12 in which the solid-liquid interface exceeded 20 degrees of inclination at the preferred withdrawal rate. Thus, future analyses should consider solid-liquid interface curvature, 
especially when utilizing FE solidification models capable of extracting the inclination of the solidification front. An additional dendrite-morphology regime was also identified between misoriented-grain formation and axial dendrite growth.

\section{Conclusions}

A validated solidification model has been applied to a range of alloys and casting conditions and has demonstrated that the position of the solid-liquid interface relative to the transition zone in the furnace can be used as a metric to identify a preferred process condition. The metric is fundamentally based on local thermal conditions at the surface of the casting, specifically the axial thermal gradient. This metric (1) indirectly accounts for a balance between thermal gradient and solidification-front curvature, (2) is independent of casting geometry and furnace temperature and generally insensitive to alloy, and (3) is directly quantifiable within solidification-modeling software for practical application.

Solid-liquid-interface curvature affects the dendrite morphology and can increase the propensity for misoriented-grain formation. Lateral growth has been associated with increased inclination and is a precursor to misoriented-grain formation.

Defect-formation criteria historically used to define a process window were modified to account for non-axial heat extraction and used to evaluate the solidification metric developed in this work. A new regime of dendrite morphology was identified on the processing map. The boundaries between dendrite-growth morphology regimes were adjusted to account for non-axial heat extraction; the critical $\mathrm{G} / \mathrm{V}$ value for the presence of nucleated grains has been adjusted to $1000{ }^{\circ} \mathrm{C}-\mathrm{s}-\mathrm{cm}^{-2}$, while the critical G/V value for the onset of lateral growth has been defined as $3500{ }^{\circ} \mathrm{C}$ $\mathrm{s}-\mathrm{cm}^{-2}$. The preferred process conditions defined by the proposed solidification metric provided thermal conditions that were within the recommended bounds of the classical solidification maps.

A model has been validated for the directional-solidification process over a broad range of thermal conditions and casting geometries. A new optimization strategy has been developed and verified on two different case histories, encompassing a range of casting geometries and withdrawal rates for two different alloys. The optimization strategy was also applied to a turbine-blade geometry, enabling identification of a time-dependent withdrawal rate that provides for maximum axial thermal gradient by maintaining constant solid-liquid interface position in the transition region of the furnace.

\section{Acknowledgements}

The authors would like to thank General Electric Company for their support in providing the airfoil model geometry.

\section{References}

1. S. Erickson, W.A. Owczarski and P.M. Curran, Metal Progress, 99 (1971) 58.

2. J.G. Tschinkel, A.F. Giamei and B.H. Kearn, "Apparatus for Casting of Directionally Solidified Articles," (U.S. Patent No 3,763,926), 1973

3. G.B. Stroganov, A.V. Logunov, V.V Gerasimov and E.L. Kats, Russian Casting Production, 12 (1983) 20.
4. M. Konter and M. Thumann Journal of Materials Processing Technology, 117 (2001) 386-390.

5. M. Konter, E. Kats and N. Hofmann, Superalloys 2000, eds. T.M. Pollock, R.D. Kissinger, R.R. Bowman, K.A. Green, M. McLean, S. Olson and J.J. Schirra (The Mineral, Metals \& Materials Society, 2000) 189-200.

6. A.J. Elliott, "Directional Solidification of Large Cross-Section Ni-Base Superalloy Castings via Liquid Metal Cooling," (Ph.D. thesis, University of Michigan, 2005).

7. A.J. Elliott, S. Tin, W.T. King, S.-C. Huang, M.F.X. Gigliotti and T.M. Pollock, Metall and Mater Trans A, 35 (2004) 32213231.

8. A.J. Elliott, G.B. Karney, M.F.X. Gigliotti and T.M. Pollock, Superalloys 2004, eds. K.A. Green, T.M. Pollock, H. Harada, T.E. Howson, R.C. Reed, J.J. Schirra and S. Walston (The Minerals, Metals \& Materials Society, 2004) 421-430.

9. J. Grossmann, J. Preuhs, W. Esser and R.F. Singer, Proceedings of the 1999 International Symposium on Liquid Metal Processing and Casting, eds. A. Mitchell, L. Ridgway and M. Baldwin (The Minerals, Metals \& Materials Society, 1999) 31-40.

10. M. Lamm and R.F. Singer, Metall and Mater Trans A, 38 (2007) 1177.

11. F. Hugo, U. Betz and J. Ren, Proceedings of the 1999 International Symposium on Liquid Metal Processing and Casting, eds. A. Mitchell, L. Ridgway and M. Baldwin (The Minerals, Metals and Materials Society, 1999) 16-30.

12. A. Kermanpur, N. Varahram, P. Davami and M. Rappaz, Metall and Mater Trans B, 31 (2000) 1293-1304.

13. J.D. Miller and T.M. Pollock, Metall and Mater Trans A, in press.

14. J.D. Miller and T.M. Pollock, Proceedings of the 2009 International Symposium on Liquid Metal Processing and Casting, ed. P.D. Lee, A. Mitchell and R. Williamson (The Minerals, Metals \& Materials Society, 2009) 119-126.

15. J. D. Miller, Heat Extraction and Dendritic Growth during Directional Solidification of Single-Crystal Nickel-base Superalloys, (Ph.D. thesis, University of Michigan, 2011).

16. C.L. Brundidge, J.D. Miller and T.M. Pollock, Metall and Mater Trans A, 42 (2011) 2723-2732.

17. C.L. Brundidge, D. van Drasek, B. Wang and T.M. Pollock, Metall and Mater Trans A, in press.

18. T.M. Pollock and W.H. Murphy, Metall Trans A, 27 (1996) 1081-1094.

19. J.D. Hunt, Solidification and Casting of Metals, ed. J.D. Hunt (London: The Metals Society, 1979), 3-9.

20. W. Kurz and D.J. Fisher, Fundamentals of Solidification, $4^{\text {th }}$ rev. ed., Enfield, NH: Trans Tech Publications, 1998.

21. M. McLean, Directionally Solidified Materials for High Temperature Service, London: The Metal Society, 1983.

22. M.C. Flemings, Solidification Processing, New York, NY: McGraw-Hill, 1974.

23. A.J. Elliott and T.M. Pollock, Metall and Mater Trans A, 38 (2007) 871-882.

24. T.J. Fitzgerald and R.F. Singer, Metall Trans A, 28 (1997) 1377-1383. 